PROCEEDINGS OF THE

AMERICAN MATHEMATICAL SOCIETY

Volume 127, Number 10, Pages 2871-2881

S 0002-9939(99)04850-9

Article electronically published on April 23, 1999

\title{
PSEUDODIFFERENTIAL CALCULUS ON MANIFOLDS WITH CORNERS AND GROUPOIDS
}

\author{
BERTRAND MONTHUBERT
}

(Communicated by Christopher D. Sogge)

\begin{abstract}
We build a longitudinally smooth, differentiable groupoid associated to any manifold with corners. The pseudodifferential calculus on this groupoid coincides with the pseudodifferential calculus of Melrose (also called $b$-calculus). We also define an algebra of rapidly decreasing functions on this groupoid; it contains the kernels of the smoothing operators of the (small) $b$-calculus.
\end{abstract}

\section{INTRODUCTION}

Let $X$ be a $C^{\infty}$ manifold modelled on $\mathbb{R}_{+}^{n}$; for any point $m \in X$ the number of zero coordinates is called the codimension of $m$.

An open face $\stackrel{\circ}{F}$ is a connected component of a set of points of the same codimension. The closure $F$ of an open face is called a face.

Following R. Melrose (see [4]), $X$ is said to be a manifold with corners if every hyperface is embedded, more precisely if for every hyperface $F$ there exists a definition function $\rho_{F} \geq 0$ on $X$, which vanishes on $F$ only, with $\mathrm{d} \rho_{F} \neq 0$ on $F$, and such that in every intersection of several hyperfaces the corresponding $\mathrm{d} \rho_{F}$ are independent.

In [4], R. Melrose introduced a pseudodifferential calculus on manifolds with boundary, and more generally with corners. Through the "b-stretched product" construction, he defined a space of pseudodifferential operators adapted to boundary problems, the $b$-calculus.

It appears in the work of Alain Connes (see [2]) that a pseudodifferential calculus is naturally associated to any Lie groupoid. The (usual) pseudodifferential calculus on a smooth manifold without boundary corresponds to the Lie groupoid $M \times M$. Moreover, introducing Connes' tangent groupoid helps us to better understand Atiyah-Singer's index theorem ([1]). Pursuing these ideas, we have made this construction explicit for any differentiable groupoid longitudinally smooth in [6].

The goal of this article is to show that Melrose's $b$-calculus is the pseudodifferential calculus on a suitable groupoid. To a manifold with corners $X$, we associate a

Received by the editors September 16, 1997 and, in revised form, December 3, 1997.

1991 Mathematics Subject Classification. Primary 35S15, 47G30, 22A22; Secondary 46L80, $19 \mathrm{~K} 56$.

Key words and phrases. Pseudodifferential calculus, manifolds with corners, groupoids, $C^{*}-$ algebras.

(C)1999 American Mathematical Society 
differentiable groupoid $\Gamma(X)$, which is longitudinally smooth, and we show that the pseudodifferential calculus on this groupoid is precisely the $b$-calculus. We also give a definition of rapidly decreasing functions on $G$ (with respect to a proper homomorphism of groupoids with values in $\mathbb{R}^{p}$ ), which allows us to consider additional smoothing operators.

Our construction generalizes straight forwardly to certain groupoids with corners (the groupoid $\Gamma(X)$ above corresponds to the groupoid $M \times M$ associated to a manifold without boundary $M)$.

We begin by introducing, in paragraph 2, some additional material about pseudodifferential calculus on a differentiable groupoid, the main ingredient being a Schwartz space on a groupoid associated with a proper homomorphism with values in $\mathbb{R}^{p}$.

In paragraph 3, we build the groupoid associated to a smooth manifold, with respect to a submanifold of codimension 1 . Then we generalize to a transverse family of submanifolds of codimension 1 .

Finally, in paragraph 4 we apply the results of the previous sections to the context of a manifold with corners. We show that the $b$-calculus coincides with the pseudodifferential calculus on the groupoid associated to the manifold.

I wish to thank François Pierrot, Michael Pimsner and Georges Skandalis for helpful discussions.

Remark. Some of these results have been independently obtained by V. Nistor, A. Weinstein and P. Xu (see [7]). A. Weinstein has stated the problem in [8].

\section{Pseudodifferential Calculus on differentiable Groupoids}

2.1. Schwartz kernels associated to pseudodifferential operators on a differentiable groupoid. Let $G$ be a longitudinally smooth, differentiable groupoid, that is to say, a groupoid together with a structure of $C^{\infty}$ manifold with corners on $G, G^{(0)}$ being a $C^{\infty}$ submanifold (with corners) of $G$, such that the fibers $G^{x}=r^{-1}(x), x \in G^{(0)}$ are smooth manifolds, the range and source maps, $r$ and $s$, are submersions, and the composition is $C^{\infty}$.

In [6] we showed that for any longitudinally smooth, differentiable groupoid, one can build a pseudodifferential calculus.

We are going to give an equivalent definition, in terms of Schwartz kernels, analogous to the definition of Hörmander ([3]) in the case of pseudodifferential operators on a smooth manifold.

We define the Lie algebroid associated to a longitudinally smooth, differentiable groupoid $G$, denoted by $\mathcal{A}(G)$, as the normal bundle of $G^{(0)}$ in $G, N_{\gamma} G^{(0)}=$ $T_{\gamma} G / T_{\gamma} G^{(0)}$. It is canonically isomorphic to the restriction to the set of units of $G$ of the kernel of the morphism $\mathrm{d} s$ (or $\mathrm{d} r): T G \rightarrow T G^{(0)}$; the sections of $\mathcal{A}(G)$ are the vector fields $v$ on $G$ which are left-invariant and such that $\mathrm{d} s(v(\gamma))=0, \forall \gamma \in G$.

The vector bundle of half-densities $\Omega^{\frac{1}{2}}$ is the line bundle over $G$ whose fiber in $\gamma \in G$ is the linear space of maps

$$
\rho: \Lambda^{k} T_{\gamma} G^{r(\gamma)} \otimes \Lambda^{k} T_{\gamma} G_{s(\gamma)} \rightarrow \mathbb{C}
$$

such that $\rho(\lambda \nu)=|\lambda|^{1 / 2} \rho(\nu), \forall \lambda \in \mathbb{R}$. Here, $k$ is the dimension of the fibers $G^{r(\gamma)}$ and $G_{s(\gamma)}$. This bundle is trivial: a choice of a metric trivializes it. 
Let $\varphi$ be a diffeomorphism from a (tubular) neighborhood $O$ of $G^{(0)}$ in $G$ to $\mathcal{A}(G)$, such that the following diagram commutes:

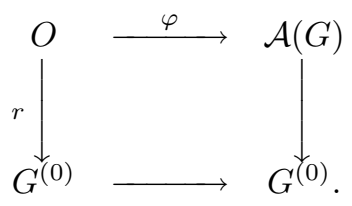

Moreover, we assume that $\varphi(\gamma)=0 \Longleftrightarrow \gamma \in G^{(0)}$, and that its differential is the isomorphim $T_{x} G^{x} \simeq N_{x} G^{(0)}$.

Definition 2.1. The space of order $m$ pseudodifferential kernels on a groupoid $G$ is the space $I^{m}\left(G, G^{(0)} ; \Omega^{\frac{1}{2}}\right)$ of distributional sections $K$ on $G$ with values in $\Omega^{\frac{1}{2}}$ which are $C^{\infty}$ outside $G^{(0)}$, and given by an oscillatory integral in a neighborhood of $G^{(0)}$ inside $O$,

$$
K=(2 \pi)^{-n} \int_{\mathcal{A}_{r(\gamma)}(G)^{*}} e^{i\langle\varphi(\gamma), \xi\rangle} a(\gamma, \xi) \mathrm{d} \xi,
$$

where $a$ is a polyhomogeneous symbol of order $m$ with values in $\Omega^{\frac{1}{2}}$.

We denote by $I^{\infty}\left(G, G^{(0)} ; \Omega^{\frac{1}{2}}\right)$ the space of pseudodifferential kernels of any order $\left(I^{\infty}\left(G, G^{(0)} ; \Omega^{\frac{1}{2}}\right)=\bigcup_{m \in \mathbb{Z}} I^{m}\left(G, G^{(0)} ; \Omega^{\frac{1}{2}}\right)\right)$.

We remark that smooth sections of $\mathcal{A}(G)$ are in $I^{1}\left(G, G^{(0)} ; \Omega^{\frac{1}{2}}\right)$. Of course, one could also choose more general symbols, like symbols of type $(\rho, \delta)$.

There is a (convolution) pairing $I^{\infty} \times C_{c}^{\infty} \rightarrow C^{\infty}$. $I^{\infty}$ is not an algebra, but $I_{c}^{\infty}\left(G, G^{(0)} ; \Omega^{\frac{1}{2}}\right)$, the space of compactly supported kernels of pseudodifferential operators, is an involutive algebra. Moreover, the convolution of an element in $I_{c}^{\infty}$ and an element of $I^{\infty}$ is an element of $I^{\infty}$.

Remark. The definition above is equivalent to that of [6], definition 2.2, where we considered the pseudodifferential operators on $G$ as $G$-equivariant families of operators on the fibers $G_{u}$.

It seems that the definitions in [7] are equivalent to the ones above. They have been obtained independently.

2.2. Rapidly decreasing functions. We have seen that Schwartz kernels of pseudodifferential operators are smooth outside the diagonal. Thus, outside a neighborhood of the diagonal, we only work with $C^{\infty}$ functions.

To obtain an algebra, we ask a priori that the functions be compactly supported. However, if we are given a proper $C^{\infty} \operatorname{map}, \varphi: G \rightarrow \mathbb{R}^{p}$, which is in addition a morphism of groupoids (in the sense that $\varphi\left(\gamma_{1} \gamma_{2}\right)=\varphi\left(\gamma_{1}\right)+\varphi\left(\gamma_{2}\right)$ ), we can then define a notion of rapidly decreasing functions on $G$. We remark that such a function does not necessarily exist; for instance, its existence immediately implies that $G^{(0)} \subset \varphi^{-1}(0)$ is compact.

If we are given such a morphism $\varphi$, we can define the space

$$
\mathcal{S}^{0}=\left\{f \in C^{\infty}(G), \forall P \in \mathbb{C}\left[X_{1}, \ldots, X_{p}\right], \sup _{\gamma \in G}\|P(\varphi(\gamma)) f(\gamma)\|<\infty\right\} .
$$

Definition 2.2. The space $\mathcal{S}(G)$ of rapidly decreasing functions on $G$, or Schwartz space on $G$, is the subspace of $\mathcal{S}^{0}$ of functions such that

$$
\forall \kappa \in I_{c}^{\infty}\left(G, G^{(0)} ; \Omega^{\frac{1}{2}}\right), \kappa * f \in \mathcal{S}^{0}, f * \kappa \in \mathcal{S}^{0} .
$$


One can easily check that $\mathcal{S}(G)$ is a subalgebra of $C^{*}(G)$.

We note that to define $\mathcal{S}(G)$ it is actually enough to consider the action of differential operators.

Thus, we can define, in this context, a pseudodifferential calculus where functions are taken in $\mathcal{S}(G)$, not only in $C_{c}^{\infty}(G)$.

Remark. This definition coincides with the usual one in the case where $G$ is $\mathbb{R}^{n}$, considered as a Lie group (whence a groupoid with $G^{(0)}$ a point).

The kernels of operators that we consider are sums of kernels of operators with compact support and of rapidly decreasing functions on $G$; they form an algebra $I_{s}^{\infty}\left(G, G^{(0)} ; \Omega^{\frac{1}{2}}\right)$ for the convolution product.

\section{The GRoupoid ASSOCIATED TO A TRANSVERSE FAMILY OF CODIMENSION 1 SUBMANIFOLDS}

3.1. The puff of a codimension 1 submanifold. Let $M$ be a manifold, and $V \subset M$ be a submanifold of $M$ of codimension 1 . We can define the normal bundle to $V$ in $M$, that we denote by $N V$, as the quotient $T M / T V$ (for $x \in V, N_{x} V=$ $\left.T_{x} M / T_{x} V\right)$.

We denote by $\mathbf{G}(V)$ the groupoid composed of the disjoint union of $M \backslash V \times M \backslash V$ and of the fiber bundle $\widetilde{V \times V}$ on $V \times V$ of the isomorphisms from $N_{y} V$ to $N_{x} V$, glued together in the following way:

$$
M \backslash V)^{2} \ni\left(x_{n}, y_{n}\right) \rightarrow(x, y, \alpha) \in \widetilde{V \times V} \Longleftrightarrow\left\{\begin{array}{l}
x_{n} \rightarrow x, y_{n} \rightarrow y \\
p_{x}\left(x_{n}-x\right)=\alpha\left(p_{y}\left(y_{n}-y\right)\right) \\
+o\left(p_{y}\left(y_{n}-y\right)\right)
\end{array}\right.
$$

where $p_{x}$ is the projection of $T_{x} M$ on the factor $N_{x} V$.

The groupoid structure on $M \backslash V \times M \backslash V$ is that of the groupoid of the trivial equivalence relation, and on $\widetilde{V \times V}$ the source and range maps, and the composition are defined as:

$$
\left\{\begin{array}{l}
r(x, y, \alpha)=x \\
s(x, y, \alpha)=y \\
(x, y, \alpha)\left(y, z, \alpha^{\prime}\right)=\left(x, z, \alpha \circ \alpha^{\prime}\right) .
\end{array}\right.
$$

The differentiable manifold structure is given by the following charts. On $M \backslash V \times$ $M \backslash V$ the structure is given by that of $M$.

Now, for $i=1,2$, let $\varphi_{i}$ be a diffeomorphism from an open $\Omega_{i}$ of $M$ to $\left(V \cap \Omega_{i}\right) \times \mathbb{R}$, such that if $x \in V \cap \Omega_{i}, \varphi_{i}(x)=(x, 0)$. The $\varphi_{i}$ determine a trivialisation of $N V$ over $V \cap \Omega_{i}$.

Then $W_{\varphi_{1}, \varphi_{2}}=\left(V \cap \Omega_{1}\right) \times\left(V \cap \Omega_{2}\right) \times \mathbb{R} \times \mathbb{R}^{*}$ defines a chart of $\mathbf{G}(V)$ by $\psi(x, y, t, \lambda)=\varphi_{1}^{-1}(y, t) \times \varphi_{2}^{-1}(x, \lambda t)$ if $t \neq 0$, and by $\psi(x, y, 0, \lambda)=(x, y, \alpha)$ where $\alpha$ is the isomorphism from $N_{y} V$ to $N_{x} V$ which, in the trivialisations given by the $\varphi_{i}$, is the dilation of rate $\lambda$.

It is clear that $\mathbf{G}(V)$ is a Lie groupoid.

In addition, the groupoid structure being given by $r \oplus s(x, y, t, \alpha)=(x, y, t, \alpha t)$, we have $d(r \oplus s)(x, y, t, \alpha)(\xi, \eta, \tau, \iota)=(\xi, \eta, \tau, \alpha \tau+t \iota)$ and in $t=0$ (that is to say, on $\left.V^{2}\right), d(r \oplus s)(x, y, 0, \alpha)(\xi, \eta, \tau, \iota)=(\xi, \eta, \tau, \alpha \tau)$. Thus, $\forall \gamma \in \widetilde{V \times V}, d(r \oplus s)\left(T_{\gamma} \mathbf{G}(V)\right.$ is the hyperplane of $T_{r \oplus s(\gamma)} G^{(0)^{2}}$ defined by the equation $\pi_{1}(\xi)=\alpha\left(\pi_{2}(\xi)\right)$, where $\pi_{2}$ 
is the projection of the second coordinate of $\xi$ on $N_{s(\gamma)}(V)$, and $\pi_{1}$ is the projection of the first coordinate of $\xi$ on $N_{r(\gamma)}(V)$.

Two particular situations can be stressed right now:

a) if $V$ is transversally oriented.

We remark that in this case the normal bundle can be trivialised, the bundle $\widetilde{V \times V}$ being trivialised in $V \times V \times \mathbb{R}^{*}$.

Moreover, the normal bundle can be decomposed into a positive part and a negative part. Thus, it is possible to restrict the bundle $\widetilde{V \times V}$ to the isomorphisms which preserve each subbundle. We denote by $\mathbf{G}(V)^{+}$the groupoid obtained; it is open in $\mathbf{G}(V)$. By using a tubular neighborhood of $V$ in $M$, we can describe $\mathbf{G}(V)$ with two charts: $M \backslash V \times M \backslash V$ and $V \times V \times \mathbb{R} \times \mathbb{R}^{*}$.

b) if $V$ splits $M$ into two parts.

In this context, we can restrict $\mathbf{G}(V)$ by keeping only $M^{+} \times M^{+} \cup M^{-} \times$ $M^{-}$, glued with the bundle of the isomorphisms in $\widetilde{V \times V}$ which preserve each subbundle. We denote by $\mathbf{G}\left(V_{+}\right)$the groupoid obtained; it is open and closed in $\mathbf{G}(V)$.

If we provide a trivialisation of $N(V), \mathbf{G}\left(V_{+}\right)$is the union of $M_{+} \backslash V \times$ $M_{+} \backslash V \cup M_{-} \backslash V \times M_{-} \backslash V$ and $V \times V \times \mathbb{R}_{+}^{*}$.

3.2. Puff of a transverse family of codimension 1 submanifolds. If we consider several codimension 1 submanifolds of $M$, we can make a similar construction provided certain conditions are fulfilled. First, recall a few facts about transversality and fiber products.

Let $X_{1}, \ldots, X_{n}, Y$ be a family of $C^{\infty}$ manifolds. A family of $C^{\infty}$ morphisms $\varphi_{i}: X_{i} \rightarrow Y$ is called transverse if, for any family $\left(x_{i}\right)_{1 \leq i \leq n} \in \Pi_{1 \leq i \leq n} X_{i}$ such that $\exists y \in Y, \forall i, \varphi_{i}\left(x_{i}\right)=y$, the orthogonals (in $T_{y}^{*} Y$ ) of the spaces $\mathrm{d} \varphi_{i}\left(T_{x_{i}} X_{i}\right)$ are in direct sum.

Under this condition, the fiber product of the $X_{i}$ over $Y$ is a $C^{\infty}$ submanifold of $\Pi_{1 \leq i \leq n} X_{i}$.

Besides, a family of submanifolds will be called transverse if the inclusions of these submanifolds are transverse.

Definition 3.1. We call decoupage a manifold $M$ together with a finite family $\left(V_{i}\right)_{i \in I}$ of codimension 1 submanifolds such that $\forall J \subset I$, the family of inclusions of $V_{j}, j \in J$, is transverse.

From now on we denote $V_{J}=\bigcap_{i \in J} V_{i}$, for $J \subset I$.

An important consequence of this definition is the following proposition:

Proposition 3.1. Let $\left(M,\left(V_{i}\right)_{i \in I}\right)$ be a decoupage. Then the differentiable morphisms $r \oplus s: \mathbf{G}\left(V_{i}\right) \rightarrow M^{2}$ are transverse.

An immediate corollary of the proposition above is that the fiber product of the $G\left(V_{i}\right)$ over $M^{2}$ is a $C^{\infty}$ manifold.

Definition 3.2. Let $\mathcal{E}=\left(M,\left(V_{i}\right)_{i \in I}\right)$ be a decoupage. The puff of the decoupage in $M^{2}$, denoted by $\mathbf{G}(\mathcal{E})$, is the fiber product of the morphisms $r \oplus s: \mathbf{G}\left(V_{i}\right) \rightarrow M^{2}$.

It is a subgroupoid and a submanifold of $\prod_{i \in I} \mathbf{G}\left(V_{i}\right)$; thus it is a Lie groupoid.

It is clear to that point that every submanifold $V_{J}$ is saturated in $\mathbf{G}(\mathcal{E})$, that is to say, that every element of $\mathbf{G}(\mathcal{E})$ whose source is in $V_{J}$ has its range in $V_{J}$. The 
orbits of $\mathbf{G}(\mathcal{E})$ are

$$
\stackrel{\circ}{F_{J}}=\left(\bigcap_{i \in J} V_{i}\right) \cap\left(\bigcap_{i \notin J} M \backslash V_{i}\right) .
$$

3.3. Puff in a transverse groupoid. We are now going to generalize the process by defining the puff of a groupoid along a transverse decoupage.

Definition 3.3. Let $G$ be a Lie groupoid; set $M=G^{(0)}$. We say that $G$ is transverse to a decoupage $\left(M,\left(V_{i}\right)_{i \in I}\right)$ if for any $J \subset I$, the differentiable morphisms $r \oplus s: G \rightarrow M^{2}$ and the inclusions $V_{i}^{2}$ in $M^{2}$, for $i \in J$, are transverse.

The following easy proposition gives some conditions equivalent to this definition:

Proposition 3.2. Let $\mathcal{E}=\left(M,\left(V_{i}\right)_{i \in I}\right)$ be a decoupage, and let $G$ be a Lie groupoid such that $G^{(0)}=M$. Then the following are equivalent:

i) $G$ is transverse to $\mathcal{E}$;

ii) for every $x \in M$, and for every $J \subset I$, the differentiable morphisms $s: G^{x} \rightarrow$ $M$ and the inclusion of $V_{J}$ in $M$ are transverse;

iii) the morphisms $r \oplus s: G \rightarrow M^{2}$ and $r \oplus s: \mathbf{G}(\mathcal{E}) \rightarrow M^{2}$ are transverse.

It is thus possible to "puff" a decoupage in $G$ :

Definition 3.4. We define the puff of the decoupage in $G$, denoted by $\langle G: \mathcal{E}\rangle$, as the fiber product:

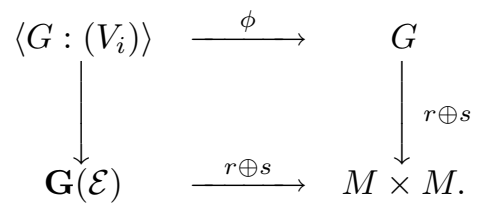

We get immediately the following proposition, due to the associativity of the fiber product:

Proposition 3.3. Let $G$ be a Lie groupoid, transverse to a decoupage $\mathcal{E}=$ $\left(M,\left(V_{1}, \ldots, V_{n}\right)\right)$. Then

$$
\langle G: \mathcal{E}\rangle=\left\langle\ldots\left\langle\left\langle G: V_{1}\right\rangle: V_{2}\right\rangle \ldots\right\rangle .
$$

In particular,

$$
\mathbf{G}(\mathcal{E})=\left\langle\ldots\left\langle\left\langle M \times M: V_{1}\right\rangle: V_{2}\right\rangle \ldots\right\rangle .
$$

We can thus obtain $\mathbf{G}(\mathcal{E})$ by puffing successively the submanifolds $V_{i}$ from $M \times$ $M$.

Corollary 3.1. For every permutation $\sigma$,

$$
\left\langle\ldots\left\langle\left\langle G: V_{1}\right\rangle: V_{2}\right\rangle \ldots\right\rangle=\left\langle\ldots\left\langle\left\langle G: V_{\sigma_{1}}\right\rangle: V_{\sigma_{2}}\right\rangle \ldots\right\rangle .
$$

We can thus puff the submanifolds following any order.

We examine what happens for a single codimension 1 submanifold.

Let $V$ be a codimension 1 submanifold of a manifold $M$, and $G$ be a Lie groupoid transverse to $V$. Then the fiber product $G^{\prime}$ of $G$ and $\mathbf{G}(V)$ over $M^{2}$ equals the union of $G_{M \backslash V}^{M \backslash V}$ and of the bundle $\widetilde{G_{V}^{V}}$ of the isomorphisms of $s^{*} N V$ to $r^{*} N V$ over $G_{V}^{V}$, both groupoids being glued together as for $\mathbf{G}(V)$.

Indeed if $\gamma^{\prime} \in G^{\prime}$, then $\gamma^{\prime}=\left(\gamma, \gamma_{V}\right)$, with $\gamma \in G, \gamma_{V} \in \mathbf{G}(V)$ such that $r \oplus$ $s(\gamma)=r \oplus s\left(\gamma_{V}\right)$. Then necessarily, $r \oplus s(\gamma) \in V^{2}$, that is to say, $\gamma \in G_{V}^{V}$, or 
$r \oplus s(\gamma) \in(M \backslash V)^{2}$, that is to say, $\gamma \in G_{M \backslash V}^{M \backslash V}$. In the first case, $\gamma^{\prime}$ is given by an isomorphism of $N_{y} V$ to $N_{x} V$, that is to say, $\gamma^{\prime} \in \widetilde{G_{V}^{V}}$. In the second case, $\gamma^{\prime}$ is completely determined by $\gamma \in G_{M \backslash V}^{M \backslash V}$, so $\gamma^{\prime} \in G_{M \backslash V}^{M \backslash V}$.

To conclude this section, we remark that the construction of the puff of a decoupage in a transverse groupoid is canonical. It does not depend, in particular, on a trivialisation of the normal bundle (in the case of a transversally oriented submanifold). However, if we are provided with such a trivialisation, we can simplify the form of the groupoid. We consider the case where the submanifolds are transversally oriented.

In this case, each normal bundle $N\left(V_{i}\right)$ can be trivialised, thanks to a definition function of $V_{i}$, denoted by $\rho_{i}$. Then the bundle $\widehat{V_{i} \times V_{i}}$ trivialises in $V_{i} \times V_{i} \times \mathbb{R}^{*}$, and

$$
G(\mathcal{E})=\bigcup_{J \subset\{1, \ldots, k\}} \bigcap_{j \in J} V_{j}^{2} \times\left(\mathbb{R}^{*}\right)^{\operatorname{card}(J)} .
$$

The topology on $G(\mathcal{E})$ is given by the convergence of the following sequences:

$$
\bigcap_{j \in J} V_{j}^{2} \times\left(\mathbb{R}^{*}\right)^{\operatorname{card}(J)} \ni\left(x_{n}, y_{n}, \lambda_{n}\right) \underset{n \rightarrow+\infty}{\longrightarrow}(x, y, \mu) \in \bigcap_{j^{\prime} \in J^{\prime}} V_{j^{\prime}}^{2} \times\left(\mathbb{R}^{*}\right)^{\operatorname{card}\left(J^{\prime}\right)},
$$

where $J^{\prime}=J \cup\left\{j_{0}\right\}$. There is convergence if and only if $x_{n} \rightarrow x, y_{n} \rightarrow y, \lambda_{j} \rightarrow \mu_{j}$, for any $j \in J$, and $\frac{\rho_{j_{0}}\left(y_{n}\right)}{\rho_{j_{0}}\left(x_{n}\right)} \rightarrow \mu_{j_{0}}$.

We obtain a groupoid: the source and range maps, and the composition are defined like this:

$$
\left\{\begin{array}{l}
r(x, y, \lambda)=x \\
s(x, y, \lambda)=y \\
(x, y, \lambda) \circ\left(y, z, \lambda^{\prime}\right)=\left(x, z, \lambda \lambda^{\prime}\right)
\end{array}\right.
$$

if $x, y$ and $z$ are in the same maximal intersection of submanifolds. If we wish to restrict to the isomorphisms which preserve each sub-bundle of the normal bundle, we obtain the same result by replacing each time $\mathbb{R}^{*}$ by $\mathbb{R}_{+}^{*}$.

Finally, we see that the puff allows us to get, from a groupoid transverse to a family of submanifolds, a groupoid which is longitudinal on these submanifolds. This is especially interesting in the context of manifolds with corners, which comes out of the case when each $V_{i}$ separates $M$ into two.

3.4. The groupoid associated to a manifold with corners. Every manifold with corners has a smooth neighborhood, thus we will take the following definition, which is equivalent to that of the introduction.

Definition 3.5. A manifold with corners $X$ is the restriction of an oriented decoupage, $\left(M,\left(V_{i}\right)_{i \in I}\right)$, where each $V_{i}$ separates $M$ into two, to the positive part of $M$. The faces are the restrictions of the intersections of the submanifolds. We say that $X$ is the positive part of this oriented decoupage.

From now on, $X$ is a compact manifold with corners.

We showed in [6] how to construct a pseudodifferential calculus far any longitudinally smooth differentiable groupoid. In the case of a manifold with corners, we cannot build a pseudodifferential calculus on the groupoid $X \times X$, because for every $x \in X,(X \times X)_{x}=X$ which is not smooth. 
However, this groupoid can be desingularized by considering the positive part of the puff of the decoupage $\mathcal{E}=\left(M,\left(V_{i}\right)_{i \in I}\right)$ for which $X$ is the positive part, following an approach due to Melrose.

Indeed, as each $V_{i}$ separates $M$ into two, we can build the fiber product of the $\mathbf{G}\left(V_{i+}\right)$, that we denote by $\mathbf{G}\left(\mathcal{E}_{+}\right)$.

We note that the positive part of the decoupage is saturated in $\mathbf{G}^{+}(\mathcal{E})$ : indeed, $\Gamma(X)=\left(\mathbf{G}(\mathcal{E})^{+}\right)_{X}^{X}$ is such that if $x \in \stackrel{\circ}{F}, \Gamma(X)^{x}=\stackrel{\circ}{F} \times\left(\mathbb{R}_{+}^{*}\right)^{\operatorname{codim}(F)}$ which is a $C^{\infty}$ manifold. Thus, it is longitudinally smooth. Also, this groupoid is independent from the decoupage considered: if $X$ embeds in another decoupage, the positive part remains unchanged, and $\Gamma(X)$ as well only depends on the positive part.

Remark. The $C^{*}$-algebra of $\Gamma(X)$ is of type I and its irreducible representations are indexed by the open faces of $X$, which correspond to subquotients isomorphic to $C_{0}\left(\mathbb{R}^{k}, \mathcal{K}_{F}^{\circ}\right)$, where $\mathcal{K}_{F}$ is the $C^{*}$-algebra of the compacts of $L^{2}(\stackrel{\circ}{F})$. Thus, it is easy to describe the spectral sequence which gives the $K$-theory of $C^{*}(\Gamma(X))$. It coincides with that of [5], and we get simple proofs of these results.

Remark. Nistor, Weinstein and $\mathrm{Xu}$ defined a groupoid, in the case of a manifold with boundary in [7], which seems to be isomorphic to $\Gamma(X)$. It was obtained independently.

\section{Pseudodifferential CAlCulus on MANifolds With CORNERS}

4.1. Schwartz space on manifolds with corners. We are now provided with all the material to build a pseudodifferential calculus with compact support on manifolds with corners.

To be able to consider, in addition to compactly supported pseudodifferential operators, some "rapidly decreasing" smoothing operators, we need to define a proper morphism of groupoids, $\Gamma(X) \rightarrow \mathbb{R}^{k}$, where $k$ is the number of submanifolds of the decoupage (that is to say, the maximal codimension of the faces of $X$ ).

First, we consider a smooth manifold $M$ together with a submanifold $V$ which splits $M$ into two. Thanks to the definition functions of $\mathrm{V}, \rho_{V}$, we can define $\psi_{V}: \mathbf{G}\left(V_{+}\right) \rightarrow \mathbb{R}$ by:

- if $\gamma \in \stackrel{\circ}{M} \times \stackrel{\circ}{M}, \psi_{V}(\gamma)=\log \left(\frac{\rho_{V}(r(\gamma))}{\rho_{V}(s(\gamma))}\right)$;

- otherwise, $\gamma=(x, y, \lambda)$ and $\varphi_{V}(\gamma)=\log (\lambda)$.

It is easy to check that $\varphi_{V}$ is a proper map. Now, for a manifold with corners $X$, we can consider a decoupage $\left(M,\left(V_{i}\right)_{i \in I}\right)$ whose positive part is $X$. The morphisms $\varphi_{V_{i}}$ then induce a proper morphism of groupoids at the level of the fiber product: $\varphi: \Gamma(X) \rightarrow \mathbb{R}^{\operatorname{card}(I)}$.

This allows us to consider a Schwartz space on $\Gamma(X)$.

Proposition 4.1. If we change the definition functions of the faces, the difference between the new morphism $\varphi^{\prime}$ and $\varphi$ is a bounded map. Thus it does not change the space of rapidly decreasing functions.

Proof. We denote by $\rho_{i}^{\prime}$ the new definition functions of the faces. Then there exists $C^{\infty}$ functions $f_{i}$ such that $\rho_{i}^{\prime}=f_{i} \rho_{i}$, with $f_{i}>0$. So $\mathrm{d} \rho_{i}^{\prime}(x)=f_{i}(x) \mathrm{d} \rho_{i}(x)$, and every isomorphism of $N_{y} V_{i}$ to $N_{x} V_{i}$ which associates $\mathrm{d} \rho_{i}^{\prime}(x)$ to $\mathrm{d} \rho_{i}^{\prime}(y)$ equals $f_{i}(x) / f_{i}(y)$ times the isomorphism which associates $\mathrm{d} \rho_{i}(x)$ to $\mathrm{d} \rho_{i}(y)$. Finally,

$$
\varphi_{i}^{\prime}(\gamma)=\log \left(f_{i}(r(\gamma)) / f_{i}(s(\gamma))\right)+\varphi_{i}(\gamma)
$$


But $f_{i}$ is continuous and does not vanish, and is defined on a compact, so the difference $\varphi_{i}^{\prime}-\varphi_{i}$ is bounded.

This allows us to build a canonical pseudodifferential calculus:

Definition 4.1. Let $X$ be a manifold with corners. The (canonical) pseudodifferential calculus on $X$ is the pseudodifferential calculus (with rapidly decreasing functions) on the groupoid $\Gamma(X)$, with respect to $\varphi$.

Remark (Pseudodifferential calculus on differentiable groupoids with corners). We can easily generalize this method to groupoids such that:

1. $G$ and $G^{(0)}$ are manifolds with corners,

2. there exists a differentiable groupoid (without corners) $G^{\prime}$ which contains $G$, such that $G^{(0)}$ admits a structure of decoupage, $G^{\prime}$ is transverse to this decoupage, and $G_{G^{(0)}}^{G^{(0)}}=G$.

We shall call differentiable groupoid with corners such a groupoid.

In this context we can define a pseudodifferential calculus by considering the puff in $G^{\prime}$ of the decoupage defined on $G^{(0)}$, then by taking its restriction to $G^{(0)}$, this groupoid being longitudinally smooth.

4.2. Relation with the $b$-calculus. For a connected manifold with boundary, the groupoid

$$
\Gamma(X)=\stackrel{\circ}{X} \times \stackrel{\circ}{X} \cup \bigcup_{1 \leq i \leq l} \partial_{i} X \times \partial_{i} X \times \mathbb{R}_{+}^{*},
$$

where $i$ indexes the boundary components, leads to the following result:

Theorem 4.1. The pseudodifferential calculus on $\Gamma(X)$ coincides with the (small) $b$-calculus of Melrose.

Proof. We recall quickly the main points of $b$-calculus ([4]). To simplify the notation, we assume that the boundary is connected.

The classical pseudodifferential calculus can be defined in terms of Schwartz kernels, by considering the pseudodifferential operators as distributional sections on $X^{2}$ with values in the bundle of half-densities, which are $C^{\infty}$ on $X^{2} \backslash \Delta$. In the $b$-calculus, the space $X^{2}$ is replaced by the $b$-stretched product, $X_{b}^{2}$ which is defined as the union of $X^{2} \backslash(\partial X)^{2}$ and of the hemispheric normal bundle of $(\partial X)^{2}$, that is to say, the quotient of the normal bundle of $(\partial X)^{2}$ by the natural action of $\mathbb{R}_{+}^{*}$. The $C^{\infty}$ structure is such that if we fix a definition function of the boundary, $\rho$, the map $\tau(x, y)=\frac{\rho(x)-\rho(y)}{\rho(x)+\rho(y)}$, defined on $\stackrel{\circ}{X}^{2}$, extends to a $C^{\infty}$ map on $X_{b}^{2}$. We also have a natural map $\beta_{b}: X_{b}^{2} \rightarrow X^{2}$.

This new space has some boundary components, in particular,

$$
\mathrm{lb}=\operatorname{cl}\left(\beta_{b}^{-1}(\partial X \times \stackrel{\circ}{X})\right)=\tau^{-1}(-1), \operatorname{rb}=\operatorname{cl}\left(\beta_{b}^{-1}(\stackrel{\circ}{X} \times \partial X)\right)=\tau^{-1}(1)
$$

(cl stands for the closure). Finally, the $b$-pseudodifferential operators on a manifold with boundary $X$ are defined by their Schwartz kernels, as distributional sections smooth outside the diagonal on the $b$-stretched product, which vanish, as well as all their derivatives, on $\mathrm{lb} \cup \mathrm{rb}$. 
Now we remark:

Lemma 4.1. Let $X$ be a manifold with boundary, and $\Gamma(X)$ be the groupoid associated to $X$. Then $\Gamma(X)$ is an open submanifold of $X_{b}^{2}$, and

$$
X_{b}^{2} \backslash \Gamma(X)=l b \cup r b .
$$

Proof. On $\Gamma(X)$, we actually have $\tau(\gamma)=\tanh (\varphi(\gamma))$, so $\Gamma(X)=\tau^{-1}(]-1,1[)$; moreover, $\tau$ is a submersion so $\Gamma(X)$ is an open submanifold of $X_{b}^{2}$, of complementary $l b \cup r b$.

We can deduce from this lemma that, as $l b \cup r b$ is disjoint from a neighborhood of $\Delta_{b}$, the pseudodifferential calculus with compact support on $\Gamma(X)$ coincides with the distributional sections on $X_{b}^{2}$, smooth outside the diagonal on the $b$-stretched product $X_{b}^{2}$, which vanish on a neighborhood of $l b \cup r b$.

Remark. The fact that compactly supported pseudodifferential operators on $\Gamma(X)$ are $b$-pseudodifferential operators was announced independently in [6] and [7].

To completely describe the (small) $b$-calculus of Melrose it remains to understand the nature of the smoothing operators, i.e., the $C^{\infty}$ functions on $X_{b}^{2}$ which vanish in the Taylor series on $l b \cup r b$.

Let $f$ be a $C^{\infty}$ function on a compact neighborhood of a point of $l b \cup r b$ adapted to $\rho$, and $g$ be its restriction to $\Gamma(X)$. If $f$ vanishes with all its derivatives on $l b \cup r b$, the Taylor formula implies that $g$ is rapidly decreasing with respect to the powers of $\tau(x, y) \pm 1$, which is equivalent to $\exp ( \pm \varphi)$. These are thus a fortiori rapidly decreasing functions with respect to $\varphi$. This means that $f \in \mathcal{S}^{0}$. Now, as the small $b$-calculus is an algebra, if $P$ is a (compactly supported) pseudodifferential operator on $\Gamma(X)$ (thus, a $b$-pseudodifferential operator), $P f$ is a smoothing operator of the $b$-calculus, and $P f \in \mathcal{S}^{0}$. Thus, $f \in \mathcal{S}$. So the smoothing operators of the $b$ calculus are in $\mathcal{S}(G)$. More precisely, they are the $C^{\infty}$ functions which are rapidly decreasing with respect to $\exp (\varphi)$.

Remark. In the case of a manifold with corners, we can build a space $X_{b}^{2}$ for each submanifold of a decoupage whose positive part is $X$. Then we can make the fiber product of these spaces, and finally consider the restriction to the positive part. It gives a $b$-stretched product for the manifold with corners.

\section{REFERENCES}

1. M. F. Atiyah and I. M. Singer, The index of elliptic operators, I, Annals of Math. 87 (1968), 484-530. MR 38:5243

2. Alain Connes, Noncommutative Geometry, Academic Press, Inc., 1994. MR 95j:46063

3. Lars Hörmander, The analysis of linear partial differential operators III: Pseudo-differential operators, Grundlehren der Mathematischen Wissenschaften [Fundamental Principles of Mathematical Sciences], vol. 274, Springer-Verlag, Berlin, 1994.

4. Richard B. Melrose, The Atiyah-Patodi-Singer index theorem, A. K. Peters, 1993. MR 96g:58180

5. Richard B. Melrose and Victor Nistor, K-theory of b-pseudodifferential operators and an $\mathbb{R}^{k}$ equivariant index theorem, Preprint.

6. Bertrand Monthubert and François Pierrot, Indice analytique et groupoïdes de lie, C.R. Acad. Sci. Paris Ser. I, 325, No. 2, 193-198 (1997). 
7. Victor Nistor, Alan Weinstein, and Ping Xu, Pseudodifferential operators on differential groupoids, Preprint.

8. A. Weinstein, Groupoids: unifying internal and external symmetry, Notices Amer. Math. Soc. 43 (1996), 744-752. MR 97f:20072

Laboratoire Emile Picard, UfR Mig, Universite Paul Sabatier, 118, route de NarBonne, 31062 Toulouse Cedex 4, France

E-mail address: monthube@picard.ups_rlse.fr

$U R L:$ http://picard.ups_rlse.fr/ monthube 